\title{
Physics of the Ocean
}

\section{Isabel Ambar, Lisbon}

(Grupo de Oceanografia, Departamento de Fisica, Universidade de Lisboa)

Essentially, a physical oceanographer has to deal with the physics of a system consisting of a non-homogeneous fluid largely covering a rotating Earth, subject to boundary actions like the winds, solar radiation and bottom stresses, and to body forces such as gravity, and the Moon's and Sun's attraction (tides): a system which exchanges momentum, heat and mass with the atmosphere. This is all highly complicated (Fig. 1). Moreover, an oceanographer is at a disadvantage relative to a physical scientist controlling the experiments in his laboratory, because his field of observation is Nature itself!

An oceanographer aims to establish a clear quantitative picture of the ocean in regard to its movements and distribution of physical properties like temperature and salt content (salinity), and to understand its behaviour in terms of physical laws so as to be able to make predictions.

Much of the descriptive knowledge of the oceans has come from the observations gathered during the large scale oceanographic expeditions of the late 1800 s and early 1900s, and also through ships' navigation log books.

Since the mid 1900s, attention has been drawn to more specific oceanographic processes, like those related to coastal upwelling ecosystems, ocean eddies, etc., and observational programmes are now organized (often as a joint effort of several countries) as experiments with a well-defined objective, instead of more or less systematic geogra- phic surveys for collecting data. Of course, this procedure requires the formulation of at least a general idea of the time and space scales of the process to be studied, in order to decide a priori on the sampling strategy.

Without pretending to be exhaustive, I shall try here to give an idea of some of the more recent fields of research in oceanography lover the past 20 years) and of the role that technological developments in physics have played in the progress of marine sciences.

\section{Simple Physical Concepts Explaining Important Ocean Processes}

Although the ocean is a rather complicated system, simple ideas in physics explain much of what we observe in it.

As a first example I refer to the model presented by Ekman in 1905 relating to a lateral boundary layer phenomenon known as coastal upwelling, whose importance can be easily assessed from the knowledge that about $50 \%$ of the world's fish catch is in areas of coastal upwelling $(0.1 \%$ of the ocean surface), like those off the west coasts of North and South America, and off the southwest and northwest coasts of A frica and Portugal.

Coastal upwelling consists essentially of the ascent of subsurface coastal waters whenever a wind blows parallel to a coastline lying on its left (right) in the northern (southern) Hemisphere (Fig. 2a). Usually, this upwelled water is rich in nutrients (phosphates, nitrates and silicates) and, when it ascends to the

Fig. 2 - Coastal upwelling and Ekman transport.
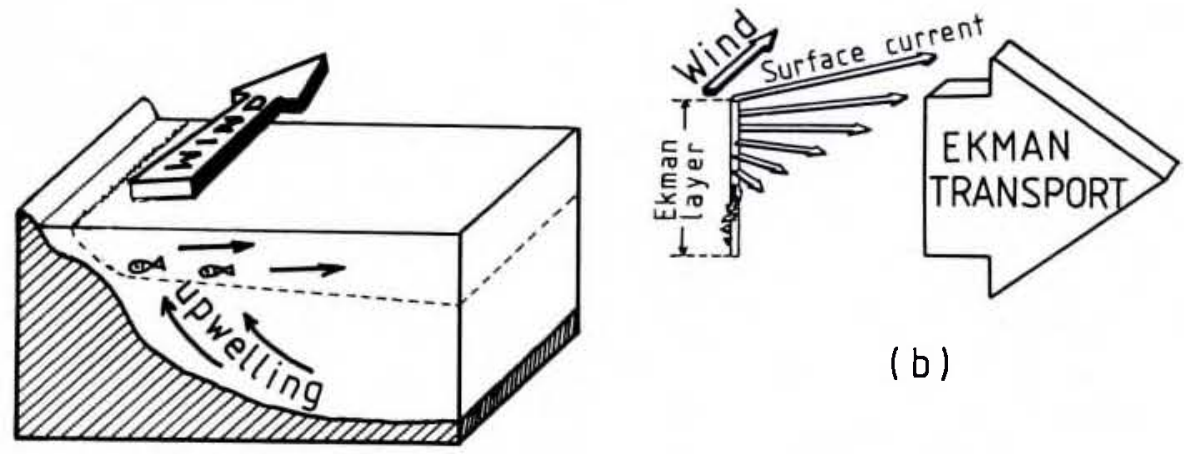

(b)

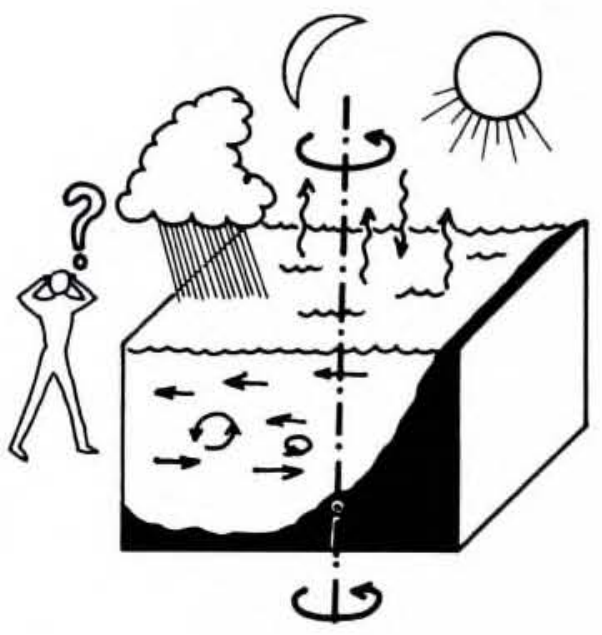

Fig. 1 - The ocean as a physical system.

euphotic (illuminated by the Sun) zone, ideal conditions (light + nutrients) are established for the growth of marine plants (phytoplankton) and hence for increased biological productivity (phyto plankton $\rightarrow$ zooplankton $\rightarrow$ fish $\rightarrow$ man).

Subsurface waters ascend to the up per levels to preserve continuity since that is the only way of replacing the surface water that is transported away from the shore by the wind. And it is this transport which is explained by Ekman's theory. From Newton's second law of motion referred to a coordinate system fixed relative to the rotating Earth, the forces acting on a mass of water can be identified as:

Real (1) + Apparent (2) which break down into pressure gradient (1.1), horizontal friction (1.2), vertical friction (1.3) and volume (1.4); Coriolis (2.1) and centrifugal (2.2).

Ekman considered a very simple situation where the only relevant terms were (1.3) and (2.1) accounting, respectively, for the vertical transfer of horizontal momentum from the wind into the surface water layers and for the deviation of horizontal flow to the right (in the northern Hemispherel owing to the Earth's rotation. The resultant of these simplified terms in Newton's law corresponds to a wind-induced horizontal motion varying with depth in the way schematicized in Fig. $2 b$ for the northern Hemisphere: the speed of flow decreases exponentially with depth and the direction makes an increasing angle to the right with respect to the wind. The depth at which the current is opposite to the surface current (the effect of the wind is then almost negligible) limits the so-called Ekman layer; its thickness is of the order of 10 $100 \mathrm{~m}$. Vertical integration of the winddriven flow for the whole Ekman layer gives the corresponding volume transport - Ekman transport — which turns 
out to be at right angles to the wind direction!

This example shows how a simple theory can sometimes explain a lot, provided that the relevant physical concepts are identified.

Another example with broad implications for ocean circulation is that of potential vorticity conservation. Vorticity is the velocity curl and is associated with the horizontal motion of a mass of water in the ocean, arising not only from its relative velocity (with vertical component $\zeta$ ) but also from the Earth's rotation in space (planetary vorticity with a vertical component $f=2 \Omega \sin \phi$, where $\Omega$ is the Earth's angular velocity and $\phi$ is the latitude). For a layer of thickness $D$ in a sea whose density is uniform, consider the combination of the continuity equation (vertically integrated for this layer) with the vorticity equation, obtained from the application of the curl operator to the equation of motion. The result is a statement of conservation of potential vorticity, defined as $(\zeta+f) / D$, for that layer in its motion, assuming that one can neglect frictional effects and the corresponding input of vorticity. The result implies, for instance, that the stretching (increased $D$ ) of a given layer moving zonally makes it acquire cyclonic (positive) rotation whereas the shrinking (decreased $D$ ) causes the layer to acquire anticyclonic (negative) rotation. This will lead to a deflection of the flow as it passes over bottom irregularities, like ridges or troughs.

This simple statement of conservation of potential vorticity, first introduced by Rossby in 1936, practically governs the large scale dynamics of the horizontal circulation and so provides a very powerful tool for the interpretation and prediction of most aspects of ocean circulation and for the tracing of water masses.

\section{Technology and Science: Some Recent Developments}

The development of electronics has contributed decisively to the fantastic improvement in oceanographic instruments and data acquisition and processing systems which has taken place over the last two decades. This, in turn, has allowed marine scientists to perceive important ocean features which had until then been out of reach.

Until about the 1960s, the distribution of water properties in the ocean was determined by using water-sampling bottles, with reversing thermometers, attached along a wire at pre-selected intervals of tens or hundreds of meters. The advent of continuous profilers of salinity or conductivity and temperature (STD or CTD systems) has allowed a resolution on much finer scales of the vertical thermohaline (temperature and salinity) variations, giving a fine structure (from 1 to several metres) and a microstructure (from millimetres to decimetres). Fig. 3 exemplifies the difference in the details obtained of the vertical structure when using a cast of classical bottles (a) or a CTD system (b).

The physics underlying the processes which lead to these smaller scale structures is no longer the same as that which applies to the large scale thermohaline perturbations, and a new field of active research has developed dealing with concepts like that of double diffusion (different molecular diffusion coefficients for heat and for salt resulting in instabilities and small scale mixing).

A second example of the contribution of modern electronics to providing a clearer view of the nature of ocean physics is the production of instruments (current meters, thermistor chains) which can be moored for long periods of time. The long-term records of currents and temperatures obtained with arrays of moored instrumentation have shown the wide spectrum of space and time variations that are encountered in the ocean, ranging from surface and internal waves (metres to kilometers; seconds to hours) to large scale circulation systems, planetary waves (hundreds to thousands of kilometers; months to years). In particular, at intermediate scales, energetic oceanic rotating systems, called mesoscale eddies, have been detected which constitute the counterparts of the synoptic cyclones and anticyclones of the atmosphere. Studies of the origin and dynamics of these eddies and of the interactions between them and both larger and smaller scale flows are still in a very incipient phase and constitute one of the major present day areas of research.

A third example of the application of modern technology to the study of ocean physics is satellite remote sensing of the sea surface using electromagnetic radiation. The great advantage of such techniques compared with the shipborne surveys lies in the possibility of covering synoptically vast areas of the globe (Fig. 4).

The types of ocean properties and processes which can be surveyed depend on the satellite sensors used. For example, microwave sensors provide information on sea surface winds (with a radar scatterometer) or on surface wave heights (using a radar altimeter), whereas infrared sensors give a mea-

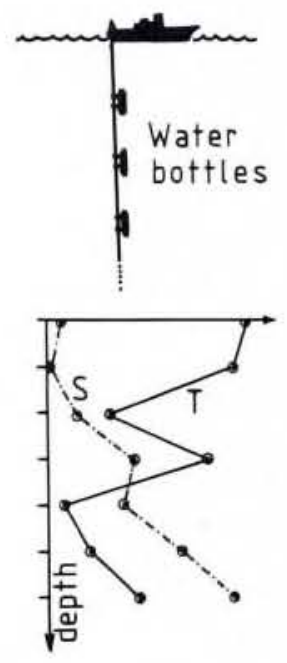

(a)

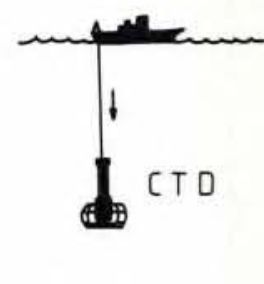

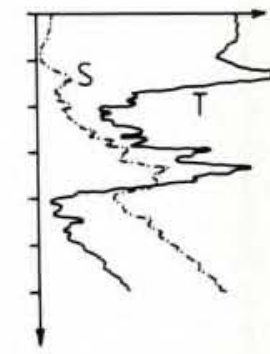

(b)
Fig. 3 - Temperature and salinity vertical profiles obtained with: (a) water bottles; (b) CTD system.

sure of the sea surface temperature and have been of great use in the identification and tracking of eddies and the detection of oceanic fronts (zones of pronounced horizontal temperature gradient) usually connected with strong biological concentrations (like those in upwelling areas).

Acoustics is a further example of a physics domain with important applications in marine science yielding instrumentation, for instance, like current meters based on the effect of water motion on the speed or frequency of sound waves (Doppler effect). Also to be noted is the new method, presently under development, of acoustic tomography which, essentially, allows us to infer ocean structures, like eddies, from the effects they produce on the sound rays originating from a given source.

\section{Ocean Climate: Present and Future Concern}

The ocean is driven by direct momentum transfer from the winds and by heating at the surface. Any change in the radiation input at the ocean surface will cause a change in the ocean heat content and, consequently, a change in sea surface temperature. This, in turn, will modify the heat flux from the ocean to the atmosphere (in the form of infrared radiation, heat conduction and latent heat of evaporation) all this may influence climate.

There is presently a great concern to evaluate the extent to which the ocean undergoes climatological fluctuations and how the dynamics of the global atmosphere-ocean system behaves on intermediate time scales (a few years). However, these studies, mainly in regard 
to the oceans, are still faced with a terrible lack of data appropriate to the study of long-period phenomena, except in the case of sea-level measurements for which there are already a reasonable number of long tide-gauge records for some oceanic areas.

The prospect of predicting climatic changes over several decades depends on the ability to build a computer model which includes the ocean and the atmosphere with their physics and circulations and the interactions between them. Such a task is still not possible owing to several factors such as the lack of a consistent data base, the insufficiency of our understanding of some ocean processes and the inadequacy of the present generation of computers which are not yet able to resolve the eddies, whose influence on the mean flow is, undoubtfully, important.

The international oceanographic community is now planning a major experiment (World Ocean Circulation Experiment, WOCE), starting in 1990, during which the necessary data base of ocean physical properties will be constituted and used for testing computer models of oceanic circulation to be applied to the research of decadal climate changes.
The aims of this project depend strongly on new techniques like satellite remote sensing, drifting buoys and acoustic tomography and their future improvement. Hopefully, the WOCE data set will be ready for use by the end of this century when the new generation of eddyresolving computers is expected to be available.

\section{Final Comments}

The development of physical oceanography as a science has passed through successive steps in which areas of physics like electronics, acoustics and optics have played an important role in the improvement of ocean observational systems and the increase of computing power. On the other hand, it must be stressed that, in a way similar to what happened three centuries ago in relation to the astronomical theory of the tides, oceanography is feeding back physics through the recently developed domain of geophysical fluid dynamics.

A new step in oceanography is about to be taken when, in the near future, new ocean circulation models, supported by a solid data base and a new computer generation, will be able to resolve the ocean eddy scales.

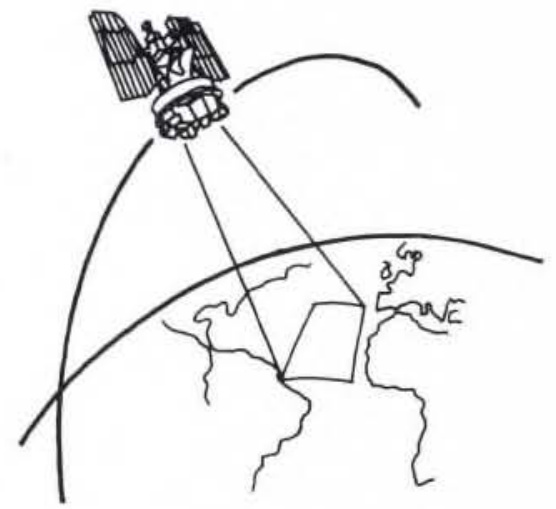

Fig. 4 - Satellite remote sensing: a new technique.

\section{SUGGESTIONS FOR FURTHER READING}

Basic level:

- Pickard G.L. and Emery W.J., Descriptive Physical Oceanography, 4th ed. (Pergamon Press) 1982, 249 pp.

- Pond S. and Pickard G.L., Introductory Dynamical Oceanography, 2nd ed. (Pergamon Press) 1983, 329 pp.

More advanced level:

- Evolution of Physical Oceanography, eds. Warren B.A. and Wunsch C. (MIT Press) $1981,623 \mathrm{pp}$.

- Gill A.E., Atmosphere-Ocean Dynamics (Academic Press) 1982, $662 \mathrm{pp}$

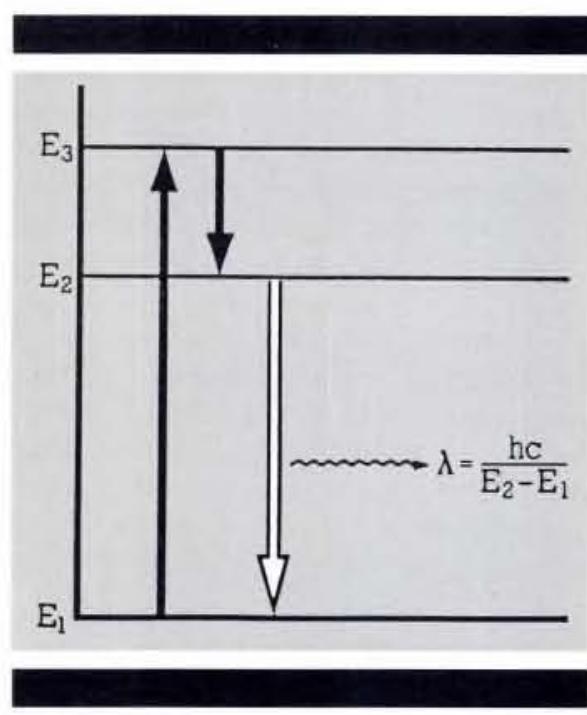

\section{PHYSICS BRIEFS $\odot$}

\section{We have 720,000 reasons* to make your physics online searches even more effective.}

In service on STN INTERNATIONAL, The Scientific \& Technical Information Network and on INKA, Information System Karlsruhe.

* Number of records in file.

For detailed information on the database, user aids and training contact:

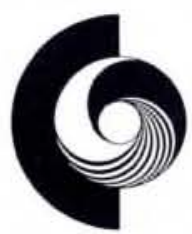

\section{Fach- nformations Physik zentrum}

FIZ Karlsruhe

D-7514 Eggenstein-Leopoldshafen 2, Federal Republic of Germany Telephone $(+49) 7247 / 82-4566$ 